\title{
Synthetic ecosystem of Escherichia coli for discovery of novel cooperative and self-adaptive algorithms
}

\author{
Kazufumi Hosoda \\ IST*, Osaka university \\ 1-5 Yamadaoka,Suita, Osaka, \\ 565-0871, Japan \\ +81-(0)6-6879-4151 \\ hosoda@ist.osaka-u.ac.jp
}

\author{
Kotaro Mori \\ FBS $^{* \star}$, Osaka university \\ 1-5 Yamadaoka,Suita, Osaka, \\ 565-0871, Japan \\ +81-(0)6-6879-4151 \\ mori_kotaro@bio.eng.osaka- \\ u.ac.jp
}

\author{
Tetsuya Yomo ${ }^{\#}$ \\ IST ${ }^{\star}$, Osaka university \\ 1-5 Yamadaoka,Suita, Osaka, \\ 565-0871, Japan \\ +81-(0)6-6879-4151 \\ Yomo@ist.osaka-u.ac.jp \\ ${ }^{\S}$ There are three more authors.
}

\begin{abstract}
Symbiosis of different biosystems is equivalent to the merge (adaptation) of different information networks. In order to understand the basis underlying symbiosis, we artificially constructed the simplest obligate and mutual symbiosis (obligate mutualism) composed of two different nutrient requiring mutants (auxotrophs) of Escherichia coli (E. coli), to find out a novel cooperative and self-adaptive algorithm. The constructed synthetic symbiosis grew with cooperative interactions and kept its growth in multiple subcultures. From the quantitative analyses, we found that $E$. coli in co-culture must keep their nutrient production rates at least 20 -fold higher than those in solo-culture, indicating the presence of cooperative and self-adaptive mechanism regulating bacterial internal conditions for the symbiosis. Furthermore, we performed "on plate" culture and succeeded in finding a typical spatial pattern of the symbiotic colony. Further analyses of this synthetic symbiosis would provide a novel algorithm for self-organization of multiple biological networks, which is likely to be applicable for information networks.
\end{abstract}

\section{Keywords}

Synthetic ecosystem, Obligate mutualism, Self-adaptive algorithm

\section{INTRODUCTION}

In general, mechanisms found in biological systems show adaptation to fluctuating and unpredictable environments. Therefore, many techniques inspired from biology have been implemented in computer science, especially in the presence of incomplete or fuzzy input data. For the foreseeable future, as information network keeps dramatic expansion now, it is a good strategy to find out novel self-adaptive algorithms from biology for applications to the vastly expanding information networks [1].

Some of large information networks can be decomposed into multiple groups of small networks, and these small networks can

\footnotetext{
Permission to make digital or hard copies of all or part of this work for personal or classroom use is granted without fee provided that copies are not made or distributed for profit or commercial advantage and that copies bear this notice and the full citation on the first page. To copy otherwise, or republish, to post on servers or to redistribute to lists, requires prior specific permission and/or a fee.

Bionetics'08, November 25-28, 2008, Hyogo, Japan.

Copyright 2008 ICST 978-963-9799-35-6.
}

have strong cooperation among each other. In biology, "symbiosis" is the phenomena where multiple organisms are interacting each other resulting in establishing self-organizing network. Here, as living organisms themselves are made of complex bio-chemical networks, the symbiosis can be considered as the merge of multiple networks. The obligate and mutual symbiosis (obligate mutualism), which is a symbiosis in which one partner is necessary for the survival of the other and vice versa, involves the strongest organism-organism (i.e., networknetwork) cooperative interaction, and thus is a good model for the information networks having network-network cooperation [2]. However, the natural obligate mutualism is very confusing because the viability of both organisms relies on the interaction of each other.

Synthetic biology is an approach to quantitatively analyze the biological systems constructed artificially. Such approach has been used to study the ecosystem, which has a high complexity [3-5]. Because of the complexity of the natural obligate mutualism, synthetic approach was used to clarify the requirement of the initial conditions for an obligate mutualism [4]. However, the reported synthetic obligate mutualism is composed of yeast, much more complex than Escherichia coli (E. coli), and any novel algorithm has not been proposed.

Previously, by constructing a genetic system of $E$. coli to analyze their adaptation to environmental changes, we proposed a novel algorithm of the biological adaptation (termed attractor selection [6]), whose concepts are now applied to the information sciences $[1,7]$. In this study, we synthesized the simplest obligate mutualism composed of E. coli (Figure 1), which is available for

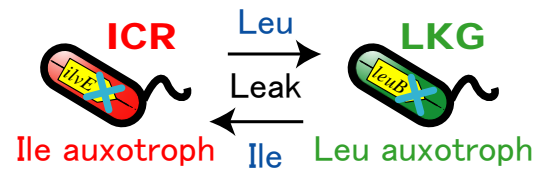

Figure 1. Scheme of our synthetic obligate mutualism.

\footnotetext{
$\S$ Yasunori Shiroguchi*, Yoshinori Yamauchi, and Akiko Kashiwagi॰

\# This author is also affiliated with $\mathrm{FBS}^{* *}$ and ERATO.

* Graduate School of Information Science and Technology

** Graduate School of Frontier Biosciences

$\checkmark$ Exploratory Research for Advanced Technology

J. Faculty of Agriculture and Life Sciences, Hirosaki University
} 
detailed quantitative analyses by using flow cytometry (FCM) [6] and microarray [8] techniques. Our purpose, as the next stage of "attracter selection," is to explore an algorithm, which explains the cooperative interactions among the "attracter selection"-based organisms resulting in an establishment of symbiosis. Considering E.coli, gene expressions in E.coli, and interaction of two strains of E.coli as a partial network in a large network, activity of network nodes in the partial network, and interaction of two partial networks, respectively, the insights obtained would contribute the information science for improving disaster tolerance of large computer networks. Here, we show the accomplishment of the synthetic obligate mutualism and some results of the analyses.

\section{RESULTS AND DISCUSSION}

\subsection{Our Synthetic Obligate Mutualism}

To create the simplest synthetic obligate mutualism, we designed the system as stated below. The system is composed of two nutrient requiring mutants (auxotrophs) of E. coli, which cooperate with each other by providing complemental nutrients. The system with a representative set of the auxotrophs is shown in Figure 1. ICR is an L-isoleucine (Ile) auxotroph lacking a gene for Ile biosynthesis (ilvE), and red-labeled by introducing a gene of red fluorescent protein (RFP). The other strain, LKG, is a Lleucine (Leu) auxotroph and green-labeled by similar genetic engineering to ICR. Both of each auxotroph can not grow without the required nutrient (Ile and Leu for ICR and LKG, respectively), however, as the required nutrients of those two auxotrophs are different, both auxotrophs can grow in the media lacking both required nutrients by providing the complemental nutrients with each other. Therefore, this synthetic system is obligate mutualism. Color labeling by fluorescent proteins not only make us possible to recognize the auxotrophs, but also can become an indicator of the bacterial condition.

\subsection{Selection of an Adequate Set of $E$. coli Auxotrophs for the Synthetic System}

We selected an adequate set of auxotrophs among twelve pairs consist of eight strains with four types of nutrient requirements (Table 1). The time courses of co-cultures in liquid media for the six pairs among twelve pairs tested are shown in Figure 2a. As the liquid medium used for cultures is minimal medium (MM63) contains no required nutrients (i.e., Ile, L-glutamine (Gln), Leu, and Uracil), all of each strain did not grow in solo-culture (data

Table 1. E. coli strains used in this study.

\begin{tabular}{cccc}
\hline Strain & Requirement & Antibiotic resistance & Color \\
\hline ICR & Ile & Chloramphenicol & Red \\
QCR & Gln & Chloramphenicol & Red \\
LCR & Leu & Chloramphenicol & Red \\
UCR & Uracil & Chloramphenicol & Red \\
IKG & Ile & Kanamycin & Green \\
QKG & Gln & Kanamycin & Green \\
LKG & Leu & Kanamycin & Green \\
UKG & Uracil & Kanamycin & Green \\
\hline
\end{tabular}

\$ We did not use antibiotics in this study.

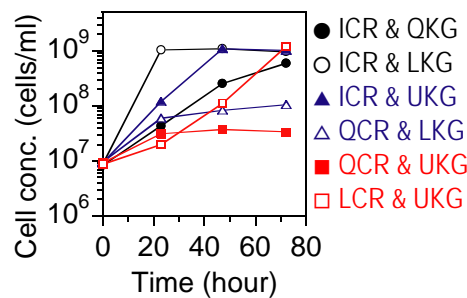

(a) Co-culture of various sets of two auxotrophs.

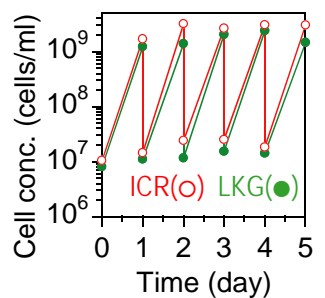

(b) Multiple subcultures of ICR and LKG.
Figure 2. Co-culture of two auxotrophs.

not shown), however, all of the co-cultures showed significant growths (Figure 2a). The time courses of the co-cultures mainly depended on the auxotrophy and showed similar results when the color markers were inversed (i.e., for instance, co-culture of LCR \& IKG showed similar results to that of ICR \& LKG). Obviously, the pair of ICR \& LKG showed the best growth and we chose the pair for analyses.

To know whether the co-culture of ICR \& LKG can keep its growth or not for extended period, we subcultured the co-culture. We tested the multiple subcultures of ICR and LKG with the passage rate of $10,1,0.1$, or $0.01 \%$, and obtained the results that those of 10 or $1 \%$ showed continuity and the others did not. The results with the passage rate $1 \%$ are shown in Figure $2 \mathrm{~b}$. These results indicate that the co-culture can be subcultured only when the passage rate is $\geq 1 \%$ (or bacterial concentration is $\geq 10^{7} / \mathrm{ml}$ ).

\subsection{Quantitative Analyses of the Synthetic System}

We formulated the population dynamics of synthetic obligate mutualism consists of ICR \& LKG and quantitatively estimated the requirements for its growth. Based on two assumptions: (1) nutrients other than Ile and Leu are sufficiently supplied in the medium and their concentration keep constant, and (2) consumptions of the nutrient supplied by the partner are mainly for growth and negligible for the others, the dynamics of the system can be formulated as

$$
\left\{\begin{array}{l}
\partial x_{1} / \partial t=m_{1} \boldsymbol{f}_{1} x_{1} \\
\partial x_{2} / \partial t=m_{2} \boldsymbol{f}_{2} x_{2} \\
\partial n_{1} / \partial t=k_{1} \boldsymbol{g}_{1} x_{1}-c_{2} m_{2} \boldsymbol{f}_{2} x_{2} \\
\partial n_{2} / \partial t=k_{2} \boldsymbol{g}_{2} x_{2}-c_{1} m_{1} \boldsymbol{f}_{1} x_{1}
\end{array}\right.
$$

where $x_{1}, x_{2}, n_{1}$, and $n_{2}$ are the concentrations of ICR, LKG, Leu, and Ile, receptively, and $m_{1}, m_{2}, k_{1}$, and $k_{2}$ are the rate constants of ICR growth, LKG growth, Leu production by ICR, and Ile production by $L K G$, respectively, in solo-culture with sufficient amino acid (Ile or Leu $\geq 1 \mu \mathrm{M}$ ), $\boldsymbol{f}_{1}, \boldsymbol{f}_{2}, \boldsymbol{g}_{1}$, and $\boldsymbol{g}_{2}$ are the function of the efficiency of ICR growth, LKG growth, Leu production by ICR, and Ile production by $\mathrm{LKG}$, respectively, in co-culture relative to those in solo-culture with sufficient amino acid (i.e., $=1$, if the rate in co-culture is the same as that in the solo-culture), and $c_{1}$ and $c_{2}$ are the conversion rates of nutrients (Ile and Leu) into $E$. coli (ICR and LKG, respectively), i.e., the amount of limiting nutrient used up in the formation of a single "standard" cell. Here, for the cells to grow continuously, all time-differentials must be $\geq$ 0 , and equations 1 can be converted as follows: 


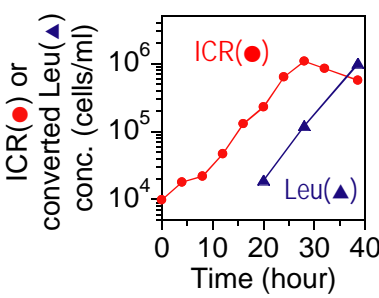

(a) Leu leak by ICR.

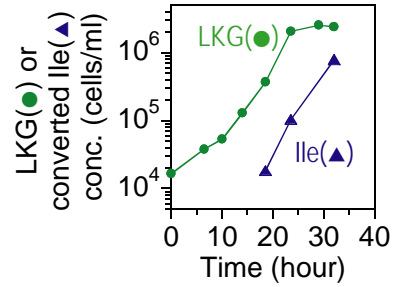

(b) Ile leak by LKG.
Figure 3. Quantitative estimation of nutrient leaks.

$$
\left\{\begin{array}{l}
\frac{k_{1}}{c_{2} m_{2}} \geq \frac{\boldsymbol{f}_{2}}{\boldsymbol{g}_{1}} \frac{x_{2}}{x_{1}} \\
\frac{k_{2}}{c_{1} m_{1}} \geq \frac{\boldsymbol{f}_{1}}{\boldsymbol{g}_{2}} \frac{x_{1}}{x_{2}}
\end{array}\right.
$$

From the experiments, we estimated the values of all constants $\left(m_{1}, m_{2}, k_{1}, k_{2}, c_{1}\right.$, and $\left.c_{2}\right)$ and the values of $\boldsymbol{f}_{1}$ and $\boldsymbol{f}_{2}$ approximated to the constants. At first, $m_{1}$ and $m_{2}$ were estimated to be 0.29 and $0.36 / \mathrm{h}$ respectively (data not shown), as slopes of the time courses in single natural logarithmic plot in solo-culture with sufficient amino acid (MM63 contains $1 \mathrm{mM}$ of Ile and Leu for ICR and LKG, respectively; see the first and second lines in equations 1 for the basis). Similarly, both $m_{1} f_{1}$ and $m_{2} f_{2}$ were determined to be $0.29 / \mathrm{h}$, as the logarithmic slopes of the time courses in co-culture (without Ile and Leu) whose sampling interval were shorter than the results shown in Figure 2a (data not shown), resulting $\boldsymbol{f}_{1}$ and $\boldsymbol{f}_{2}$ were 1.0 and 0.81 , respectively. Next, $c_{1}$ and $c_{2}$ were estimated to be 0.50 and $0.25 \mathrm{fmol} / \mathrm{cell}$, respectively, as stated below. Take $c_{1}$ for example, when $x_{1,0}$ (the initial concentration) of ICR was inoculated into a liquid medium contains $n_{2,0}$ (the initial concentration) of Ile (solo-culture), the increase of ICR should be equal to the decrease of Ile divided by $c_{1}$ (see first and fourth lines in equations 1 when $x_{2}=0$ and $f_{1}=1$ ), therefore, the final concentration of ICR and Ile are

$$
x_{1, \mathrm{f}}=x_{1,0}+n_{2,0} / c_{1}
$$

and $n_{2, \mathrm{f}}=0$, respectively. In our experiment, $x_{1, \mathrm{f}}$ and $n_{2,0}$ were indeed proportional (data not shown) and the value of $c_{1}$ as the proportionality constant was determined as stated above. The value of $c_{2}$ was determined by the same way.

Then, we determined $k_{1}$ and $k_{2}$ at 0.0057 and 0.0077 fmol.cell ${ }^{-1} \mathrm{~h}^{-1}$, respectively, as described bellow. Consider $k_{1}$ for example, the time courses of the concentrations of ICR and Leu in solo-culture $\left(x_{2}=0\right.$ and $\boldsymbol{f}_{1}=\boldsymbol{g}_{1}=1$, see first and third lines in equations 1) can be solved analytically as

$$
\left\{\begin{array}{l}
x_{1}=x_{1,0} \cdot \exp \left(m_{1} \cdot t\right) \\
\frac{n_{1}}{c_{2}}=\frac{k_{1}}{c_{2} m_{1}} \cdot x_{1,0} \cdot\left\{\exp \left(m_{1} \cdot t\right)-1\right\}
\end{array}\right.
$$

where $n_{1} / c_{2}$ represents the concentration of Leu whose unit is converted to the concentration of LKG (see the definition of $c_{2}$ shown above). The time courses of the concentration of ICR $\left(x_{1}\right)$ and the converted Leu $\left(n_{1} / c_{2}\right)$ in the solo-culture (MM63 contains $1 \mu \mathrm{M}$ of Ile) were shown in Figure $3 \mathrm{a}$. The results show that the growth rate (single logarithmic slope) of $x_{1}$ and $n_{1} / c_{2}$ is indeed almost the same, and we estimated $k_{1}$ value as stated above from the ratio of $n_{1} / c_{2}$ to $x_{1}$ (i.e., $k_{1} \cdot c_{2}^{-1} m_{1}^{-1}$ ), $c_{2}$ value, and $m_{1}$ value. The value of $k_{2}$ was determined by the same way (Figure $3 \mathrm{~b}$ ).
Table 2. Colony-forming unit (CFU) per plate .

\begin{tabular}{ccccc}
\hline $\begin{array}{c}\text { Inoculation } \\
\text { (cells/plate) }\end{array}$ & ICR 0 & ICR 10 & ICR $10^{3}$ & ICR $10^{5}$ \\
\hline LKG 0 & 0 & 0 & 0 & 0 \\
LKG 10 & 0 & 0 & 0 & 30 \\
LKG $10^{3}$ & 0 & 0 & 0 & $>1000$ \\
LKG $10^{5}$ & 0 & 31 & $>1000$ & Lawn $^{\$ \$}$ \\
\hline
\end{tabular}

\$ \$ Bacterial lawn, which is not countable as CFU.

We quantitatively considered the requirements of the growth of the system (equations 2) from the estimated values. Surprisingly, both of the constants shown in the left-hand sides of equation 2 were much less than $1\left(k_{1} \cdot C_{2}{ }^{-1} m_{2}{ }^{-1}\right.$ and $k_{2} \cdot c_{1}^{-1} m_{1}^{-1}$ were 0.064 and 0.053 , respectively) even this system consists of the best set of auxotrophs (Figure 2a), which means that any self-adaptive mechanism, regulating bacterial internal conditions for the symbiosis, can be present as described below. For simple understanding, take three approximations: (1) $x_{1} \approx x_{2}$ (see Figure 2b), (2) $\boldsymbol{f}_{1} \approx \boldsymbol{f}_{2} \approx 1$ (see above), and (3) $k_{1} \cdot c_{2}{ }^{-1} m_{2}{ }^{-1} \approx k_{2} \cdot c_{1}{ }^{-1} m_{1}{ }^{-1} \approx$ $1 / 20$ (see above), then, the requirements in equations 2 are reduced to

$$
\boldsymbol{g}_{i} \geq 20
$$

where $i$ is 1 or 2 . Recall that the functions $\boldsymbol{g}_{1}$ and $\boldsymbol{g}_{2}$ are the efficiency of the complemental nutrient productions in the coculture relative to those in solo-culture with sufficient amino acid. That is, E. coli in co-culture must keep their production rates (nutrient leaking rates) at least 20-fold higher than those in soloculture. This result suggests that any self-adaptive and cooperative mechanism of bacterial conditions was operating in our system. We will clear the mechanism by both theoretical approach and detailed experimental analysis using flow cytometry (FCM) [6] and microarray [8] techniques.

\subsection{Pattern Formation of the Symbiotic Colony on the Plate Medium}

We performed the incubation of the system on plate media to add effects of spatial information to the synthetic system. We cocultured (ICR and LKG) on MM63 agar plate $\left(9.6 \mathrm{~cm}^{2}\right)$ at various density (summarized in Table 2). The results in table 2 clearly show that the presence of both strains is necessary for the colony formation, and that almost the same numbers of colonies as the inoculated cell-numbers of fewer strains were formed on the plates where the greater strains were inoculated at $10^{5}$ cells/plate. The results indicate that there is a threshold in the bacterial density between $10^{3}$ and $10^{5}$ cells/plate for the symbiotic colony formation. These results are attributed to both the consumption and the production of nutrients as in the liquid co-culture.

We observed the synthetic colonies by fluorescent microscopy to recognize the difference of spatial characters in the colonies. Figure 4 shows the results of the observation for a typical colony on each plates inoculated at ICR and LKG are $10^{5}$ and 10 cells/plate, respectively (left), and 10 and $10^{5}$ cells/plate, respectively (right). The microscopic pictures and the fluorescent density as a function of horizontal space of the transparent blue bar in the pictures are shown at upper and lower in the figure, respectively. The fluorescent intensities (lowers) were detected 


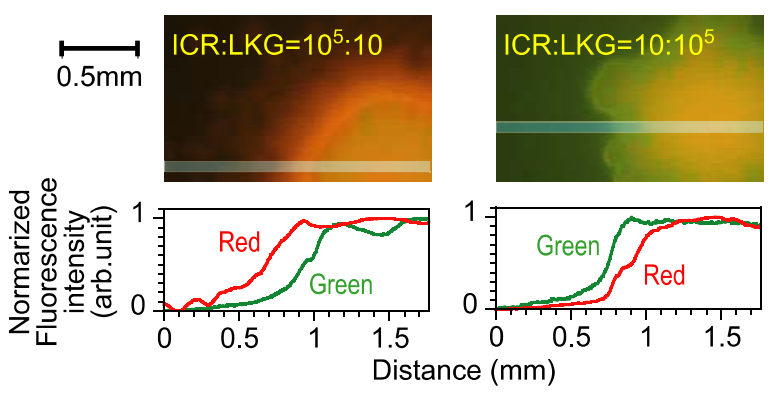

Figure 4. Microscopic observation of symbiotic colony.

separately for those of red and green using individual filter lens, and normalized to be that the maximum and minimum of the intensity become 1 and 0 , respectively. The horizontal axes of the lower graphs correspond the horizontal positions in upper pictures. The upper pictures clearly show that there is a pattern in the symbiotic colonies, which is that the outer circumferences of the colonies consist of almost a single strain whose density is greater $\left(10^{5}\right.$ cells/plate), and the lower graphs show that the width of the belt of outer circumferences are about $200 \mu \mathrm{m}$. We considered that these results are attributed to the diffusion of both nutrients and E. coli, further to the bacterial growth and the nutrient production. Color-labeled colony patterns made by a programmed intercellular interaction in a synthetic ecosystem using autoinducer, which is a signaling molecule used in quorum sensing (intercellular communications), were reported previously [9], however, the pattern observed in this study is not based on known autoinducers but based on the mutual nutrient supplement. As the observed colony pattern is likely to involve the cooperative adaptation of bacterial conditions shown in the sections for the liquid culture, it is important to analyze this "on plate" synthetic system for exploring self-adaptive algorithms of role sharing and partial grouping in biological cooperative system.

\section{CONCLUSIONS}

We constructed the simplest synthetic obligate mutualism composed of two different auxotrophs of E. coli (Figure 1). We verified that the synthetic co-culture grew with cooperative interactions and kept its growth in multiple subcultures. Moreover, from the quantitative analysis, we found that $E$. coli in co-culture must keep their nutrient production rates at least 20-fold higher than those in solo-culture, indicating the presence of self-adaptive mechanism regulating bacterial internal conditions for the symbiosis. Furthermore, we performed "on plate" culture and succeeded in finding a typical spatial pattern of the symbiotic colony. Further analyses of this synthetic symbiosis would provide a novel cooperative and self-adaptive algorithm for selforganization of multiple biological networks, which is likely to be applicable for information networks, as the next step of the individual bacterial adaptation "attractor selection", we proposed previously $[6,7]$.

\section{MATERIALS AND METHODS}

All E. coli strains used in this study are deviations of DH1 strain. Modified M63 media (MM63; contains $100 \mathrm{mM} \mathrm{KH}_{2} \mathrm{PO}_{4}, 15 \mathrm{mM}$ $\left(\mathrm{NH}_{4}\right)_{2} \mathrm{SO}_{4}, 1.8 \mu \mathrm{M} \mathrm{FeSO} \cdot 7 \mathrm{H}_{2} \mathrm{O}, 15 \mu \mathrm{M}$ Thiamine $\cdot \mathrm{HCl}, 0.2 \mathrm{mM}$ $\mathrm{MgSO}_{4}, 22.2 \mathrm{mM}$ Glucose) were basically used for all cultures in this study. For plate culture, agar was added to MM63 at 2\% $(\mathrm{w} / \mathrm{w})$. The cell concentration was determined by coulter counter (Multisizer 3, Beckman coulter) or by FCM (FC 500, Beckman coulter) using calibration beads as a standard. We used bioassay to determine Ile or Leu concentration in the culture solution.

\section{ACKNOWLEDGMENTS}

We are very grateful to Dr. Tomoaki Matsuura for fruitful discussion. This research was supported by "The Global COE (Centers of Excellence) Program" and "Special Coordination Funds for Promoting Science and Technology: Yuragi Project" of the Ministry of Education, Culture, Sports, Science, and Technology, Japan.

\section{REFERENCES}

[1] Leibnitz K, Wakamiya N, and Murata M. Biologically inspired self-adaptive multi-path routing in overlay networks. Commun. ACM 49, 2006, 62-7

[2] Wakamiya $\mathrm{N}$ and Murata M. Overlay network symbiosis: evolution and cooperation. In Proceedings of the 1st international conference on Bio inspired models of network, information and computing systems (Bionetics 2006) (Cavalese, Italy, December, 2006). Article No. 32

[3] Yamada A, Matsuyama S, Todoriki M, Kashiwagi A, Urabe I, Yomo T. Phenotypic plasticity of Escherichia coli at initial stage of symbiosis with Dictyostelium discoideum. Biosystems 92, 2008, 1-9

[4] Shou W, Ram S, Vilar JM. Synthetic cooperation in engineered yeast populations. Proc Natl Acad Sci U S A 104, 2007, 1877-82

[5] Balagadde FK, Song H, Ozaki J, Collins CH, Barnet M, et al. A synthetic Escherichia coli predator-prey ecosystem. Mol Syst Biol 4, 2008, 187

[6] Kashiwagi A, Urabe I, Kaneko K, Yomo T. Adaptive response of a gene network to environmental changes by fitness-induced attractor selection. PLOS ONE 1, 2006, e49

[7] Furusawa C, Kaneko K. A Generic Mechanism for Adaptive Growth Rate Regulation. PLoS Computational Biology 4, 2008, e3

[8] Suzuki S, Ono N, Furusawa C, Kashiwagi A, Yomo T. Experimental optimization of probe length to increase the sequence specificity of high-density oligonucleotide microarrays. BMC Genomics 8, 2007, 373

[9] Basu S, Gerchman Y, Collins CH, Arnold FH, Weiss R. A synthetic multicellular system for programmed pattern formation. Nature 434, 2005, 1130-4 